\title{
Vertebral arteries bilaterally passing through stellate (cervicothoracic) ganglion
}

\author{
B. Chaudhary' ${ }^{1}$ P.R. Tripathy², M.R. Gaikwad ${ }^{2}$ \\ ${ }^{1}$ All India Institute of Medical Science, Patna, India \\ ${ }^{2}$ All India Institute of Medical Science, Bhubaneswar, India
}

[Received: 27 August 2019; Accepted: 5 October 2019]

\begin{abstract}
Vertebral artery is a branch of the first part of subclavian artery. Vertebral artery arising from the aortic arch most commonly presents on the left side. The cervical part of sympathetic trunk is closely related to the vertebral artery in the cervical region. Though lots of variations regarding anomalous origin, course of vertebral artery is reported in the literature, here we present a rare anomaly in which vertebral artery after originating from aortic arch is passing through stellate ganglia and it enters into the transverse foramina of higher cervical vertebra (C5). Such variation should be kept in mind by anaesthetist during stellate ganglion block in order to relieve intractable pain in central nervous system lesion. Surgeons should keep this anomaly in mind during cervical spine surgery otherwise vertebral artery may get injured leading to haemorrhage. (Folia Morphol 2020; 79, 3: 621-626)
\end{abstract}

Key words: aortic arch, haemodynamic, Horner syndrome, sympathetic ganglia, vertebral artery

\section{INTRODUCTION}

In normal anatomy of aortic arch and its great vessels, vertebral artery (VA) arises from the first part of subclavian artery (SA). Numerous articles on VA are available in the literature regarding its anomalous origin, course and tortuosity, fenestration and its entry into foramen transversarium $[1,11,13,16,17]$. Here, we present a very rare anomaly in which VA are bilaterally passing through the stellate ganglion before entering into foramen transversarium with anomalous origin of left vertebral artery (LVA) from aortic arch.

The left subclavian artery (LSA) is one of the three branches that arise from aortic arch whereas the right subclavian artery (RSA) arises from the brachiocephalic trunk. For description, each SA is divided into three parts by scalenus anterior muscle [15].

Vertebral artery supplies blood to upper spinal cord, brainstem, cerebellum and posterior cerebral hemisphere [15]. It is divided into four segments. The first segment (V1) extends from its origin up to its entry into C6 transverse foramen, the second part (V2) ascends through upper five cervical transverse foramina, the third part (V3) emerges from the first cervical vertebra and the fourth part (V4) enters cranial cavity, unites with its fellow to form basilar artery [17]. The VA and corresponding vein extends vertically from the base to apex of scaleno-vertebral triangle. Medial, lateral boundaries and base of the triangle is formed by longus colli, scalenus anterior and the first part of SA, respectively. At root of the neck, the first part of vertebral artery (V1), its accompanying vein and cervical part of sympathetic trunk (CST) are contents of scaleno-vertebral triangle [3].

The CST runs up medial to the VA and presents three ganglia - superior, middle and inferior. Superior cervical ganglion (SCG) lies in front of C2 and 

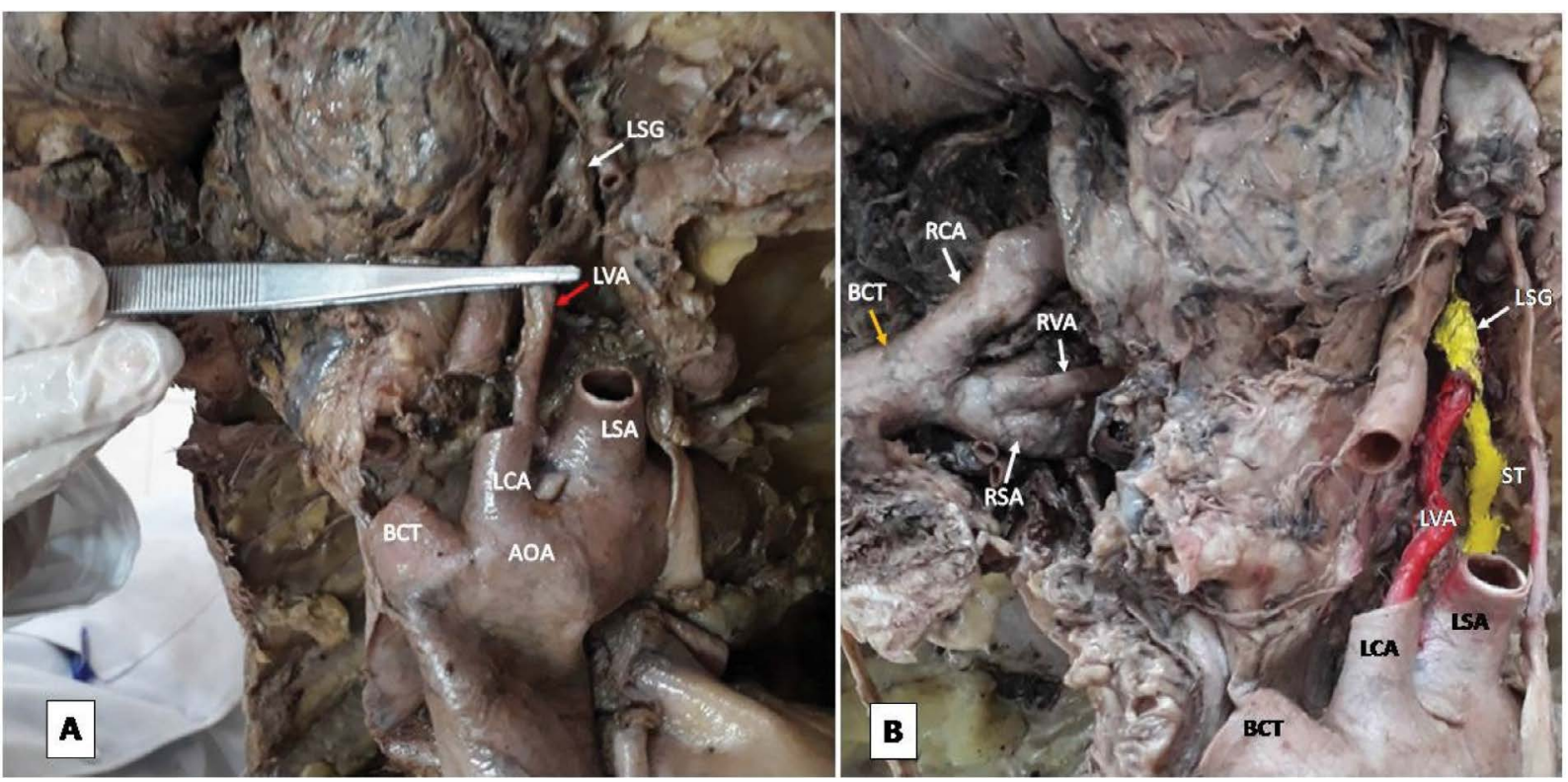

Figure 1. A. Four vessels arising from arch of aorta. Left vertebral artery (LVA; red arrow) arising from aorta is passing through left stellate ganglion (LSG; white arrow); AOA — aortic arch; BCT — brachiocephalic trunk; LCA — left common carotid artery; LSA — left subclavian artery; B. Left vertebral artery (coloured in red) is passing through left stellate ganglia (white arrow). BCT is sectioned and its proximal end reflected (yellow arrow) to show normal origin of right vertebral artery (RVA) from right subclavian artery (RSA); RCA — right common carotid artery; ST — sympathetic trunk (coloured in yellow).

C3 vertebrae. Middle cervical ganglion (MCG) lies medial to the carotid tubercle (C6 vertebra) and the inferior cervical ganglion (ICG) lies behind the commencement of vertebral artery. ICG is often fused with the first thoracic ganglion to form stellate (cervicothoracic) ganglion (SG) in front of the neck of the first rib [14].

\section{CASE REPORT}

It was an incidental finding in an embalmed formalin fixed male cadaver during routine undergraduate dissection class of thorax in Department of Anatomy. After demonstrating the boundaries and content of middle mediastinum, heart was tried to be removed by sectioning the three major vessels i.e. brachiocephalic trunk, left common carotid artery (LCA) and LSA arising from arch of aorta but some resistance was found during its removal. On observation it was found that a fourth vessel is originating from the aortic arch between the LCA and LSA (Fig.1A). Midline incision was given in the cervical region to trace the course of the fourth vessel. Skin and fascia were reflected laterally. Sternocleidomastoid muscle was transected and reflected to expose the neurovascular complex. Carotid artery, CST was identified and length and diameter of the fourth vessel was measured using digital Vernier calliper.
For microscopic anatomy of the fourth vessel, small piece of artery was dissected out from the middle of the V1 segment and was preserved in 10\% formalin solution. The tissue was processed, embedded in paraffin. Sections were taken using rotatory microtome and slide was prepared and stained with haematoxylin and eosin stain (Fig. 1H, E) to look for any pathology (Fig. 1).

\section{Observation of Gross specimen}

The fourth vessel arising from aortic arch between LCA and LSA was LVA (Fig.1A). The artery ascended and inclined towards the left for $1.5 \mathrm{~cm}$, passed through the stellate ganglia and then entered foramen transversarium of $C 5$ cervical vertebra (Fig. 1B, 2A). In present case, LVA was medial to the sympathetic trunk in its proximal part, SCG was present between $\mathrm{C} 2$ and $\mathrm{C} 3$ level, middle ganglion at the level of $\mathrm{C} 6$ transverse process and the ICG was fused to the first thoracic ganglia to form SG at the level of the first rib on either side. The right vertebral artery (RVA) had its normal origin from RSA but before entering into the foramen transversarium it RVA was also found to be passing through the SG on the right side (Fig. 2B). The average length of $\mathrm{V} 1$ segment of left and right VA was $5.46 \pm 0.15 \mathrm{~cm}$ and $2.53 \pm 0.05 \mathrm{~cm}$, respectively, whereas average diameter of left and right VA 


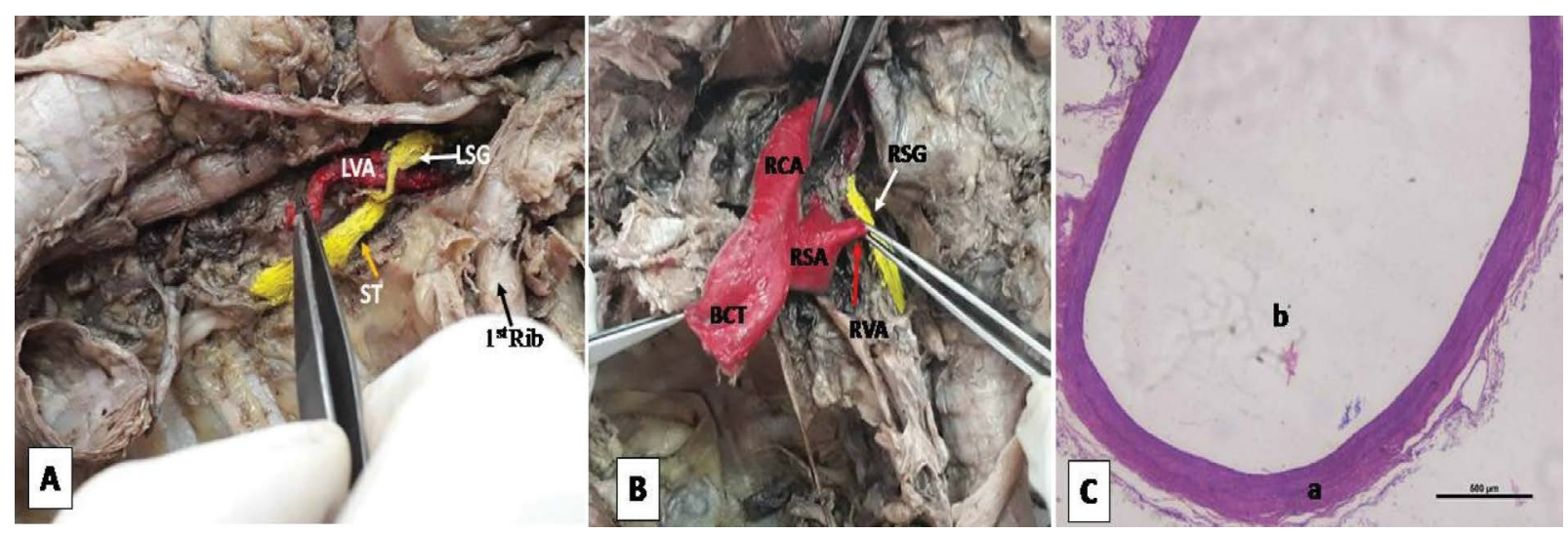

Figure 2. A. Left vertebral artery (LVA; coloured in red) is passing through left stellate ganglia (LSG; white arrow). Left stellate ganglia present at the level of the first rib; ST — sympathetic trunk (coloured in yellow); B. Right vertebral artery (RVA; red arrow) is passing through stellate ganglia on right side; BCT — brachiocephalic trunk; RCA — right common carotid artery; RSA — right subclavian artery; RSG — right stellate ganglia; C. Haematoxylin and eosin stained section of the first segment of left vertebral artery showing abundant elastic fibres and smooth muscle fibres in tunica media (a), patent lumen (b); scale bar $=500 \mu \mathrm{m}$.

was $4.23 \pm 0.05 \mathrm{~mm}$ and $4.2 \pm 0.2 \mathrm{~mm}$, respectively. The VA and CST were painted with red and yellow poster colour, respectively, and photographs were taken (Fig. 2).

\section{Observation of microscopic specimen}

Under microscope, haematoxylin and eosin stained section of artery showed presence of abundant elastic fibres and smooth muscle fibres in tunica media (Fig. 2C). Irregularity of arterial wall and separation of media was not seen. No haematoma was found. The artery was patent with no obvious occlusive disease.

\section{DISCUSSION}

Anomalous origin of VA from aortic arch has been reported previously by many authors [9, 16, 19]. Tardieu et al. [16] has reported that VA arising from aortic arch take a more medial course over the cervical vertebral bodies and it enters a transverse foramen that was more cranially located than the normal C6 entrance. According to Woraputtaporn et al. [19], in $78.6 \%$ of cases LVA of aortic origin entered the fifth cervical transverse foramina. The prevertebral part of VA of aortic origin was less protected by bone and was twice as long as RVA. Polguj et al. [10] has reported a case of extracranial duplication of VA with coexisting spontaneous dissection of internal carotid artery and Ehlers-Danlos syndrome during multidetector 64 row computed tomography and Doppler ultrasonography B-flow mode. According to the authors LVA having 2 origins from LSA fused to form a single VA which entered transverse foramen of C5. VA fenestration and duplication predisposing to ar- terial dissection results in stroke was concluded by Polguj et al. [11]. Dzierzanowski et al. [2] has analysed the morphometry of the intracranial segment of VA (V4) in context of its clinical usefulness using digital subtraction angiography and three-dimensional angio-computed tomography. The authors have reported that incidence of V4 ectasia was more and was found in the natural location of formation of saccular aneurysm [2]. Elgueta et al. [4] have reported VA commonly travels adjacent to areas targeted by the third occipital nerve procedures and rarely over the access point for atlantoaxial joint injections. Modification to existing techniques may reduce the risk of accidental VA breach during performance of cervical pain block procedure [4]. A rare variation of VA was reported by Shoja et al. [12] in which LVA after ascent in neck through the transverse foramina passed posteriorly between $\mathrm{C} 3$ and $\mathrm{C} 4$ transverse process and terminated extracranially by supplying posterior muscles of the neck. The RVA continued as basilar artery [12].

In an observational study done by Komiyama et al. [7], LVA of aortic origin was associated with higher incidence of arterial dissection than VA of subclavian artery origin (Fig. 3A). The authors have reported that there is more haemodynamic stress in the artery arising from aorta because of higher pressure and pulsation. As VA arising from aortic arch receives direct arterial pulsatile flow than the VA of subclavian origin which receives damped flow, alteration of cerebral haemodynamics occurs in VA of aortic origin. The VA of aortic origin enters at C5-6 intervertebral level. The higher entry of VA to 


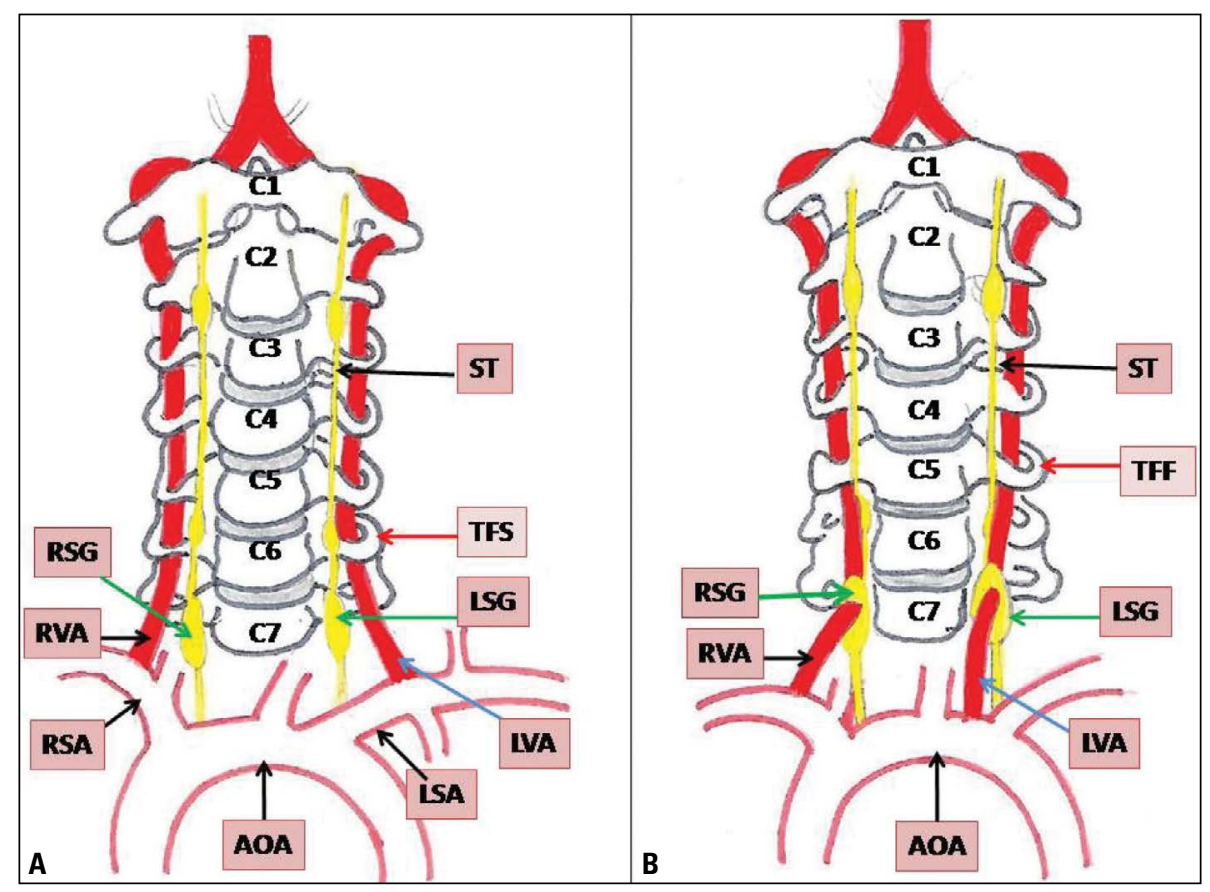

Figure 3. A. Schematic diagram showing normal origin of vertebral artery from subclavian artery entering into transverse foramina of sixth cervical vertebra (red arrow). Lower part of the first segment of vertebral artery (blue arrow) is lateral to sympathetic trunk; AOA — aortic arch; TFS — sixth cervical vertebra transverse foramen; LVA — left vertebral artery; LSA — left subclavian artery; RVA — right vertebral artery; RSA — right subclavian artery; RSG — right stellate ganglia; ST — sympathetic trunk; B. Our present case, left vertebral artery (blue arrow) of aortic origin is medial to sympathetic trunk in the proximal part and is passing through stellate ganglion (green arrow) before entering fifth cervical vertebra transverse foramen (TFF; red arrow). Right vertebral artery of subclavian origin is also passing through stellate ganglion (green arrow).

Table 1. Descriptive summary of comparison of other works related to vertebral artery anomaly

\begin{tabular}{|c|c|c|c|c|}
\hline Reference & Country & Methodology & Anomaly of VA & Accompanying vascular anomaly \\
\hline Cheng M et al. (2009) & China & $\begin{array}{l}\mathrm{CT}, \mathrm{MRI} \text {, cerebral } \\
\text { angiography, } \\
\text { preoperative DSA }\end{array}$ & $\begin{array}{c}\text { Origin of RVA from RCA } \\
\text { LVA had double origin from LSA } \\
\text { and aortic arch }\end{array}$ & $\begin{array}{l}\text { RSA arising from middle } \\
\text { of aortic arch }\end{array}$ \\
\hline Dzierzanowski J et al. (2017) & Poland & DSA, 3D-CT & $\begin{array}{l}\text { V4 segment of LVA predominant } \\
\text { over right artery }\end{array}$ & $\begin{array}{l}\text { V4 ectasia were found in natural } \\
\text { location of formation of } \\
\text { saccular aneurysm }\end{array}$ \\
\hline Elgueta MF et al. (2018) & Canada & CTA & $\begin{array}{c}\text { VA loop originating between } \mathrm{C} 1 \text { and } \mathrm{C} 2 \\
\text { travel over anterolateral aspect of inferior } \\
\text { articular pillar of } \mathrm{C} 2\end{array}$ & \\
\hline Komiyama M et al. (2001) & Japan & Angiography & LVA arising from aortic arch & $\begin{array}{l}\text { Arterial dissection of LVA of aortic origin } \\
\text { LVA entering into C5 transverse foramen }\end{array}$ \\
\hline Polguj M et al. (2013) & Poland & CTA & LVA duplication & Dissection of right internal carotid artery \\
\hline Shoja MM et al. (2006) & Iran & Surgery & $\begin{array}{l}\text { LVA passed between C3 and C4 } \\
\text { transverse process terminated } \\
\text { extracranially }\end{array}$ & RVA continued as basilar artery \\
\hline Tardieu GG et al. (2017) & Grenada & $\begin{array}{l}\text { Cadaver, CTA, } \\
\text { arteriography }\end{array}$ & LVA arising from aortic arch & $\begin{array}{l}\text { VA entered transverse foramen of } \\
\text { higher cervical vertebra }\end{array}$ \\
\hline Tetiker H et al. (2014) & Turkey & $\begin{array}{l}\text { Carotid Doppler USG, } \\
\text { vertebral artery } \\
\text { angiography }\end{array}$ & Fenestration of VA & $\begin{array}{l}\text { Atheroma plaque starting from } \\
\text { sinus caroticus extended up to } \\
\text { internal carotid artery }\end{array}$ \\
\hline Tubbs RS et al. (2016) & USA & Dry bone specimen & VA makes medial indentation at C2 & Larger caves were found on left side \\
\hline
\end{tabular}

CT — computed tomography; CTA — computed tomography angiography; DSA — digital subtraction angiography; 3D — three-dimensional; USG — ultrasonography; LVA — left vertebral artery; LSA — left subclavian artery; RCA — right common carotid artery; RVA — right vertebral artery; RSA — right subclavian artery; VA — vertebral artery 
C5 foramen transversarium may cause larger sheer stress in distal portion of the VA. The authors have reported that one elderly patient with aortic origin of LVA presented with left Horner's sign [7]. Any injury to the sympathetic trunk results in Horner's syndrome which is characterised by ptosis, enophthalmos, meiosis, and anhydrosis on the affected side [15]. In present case, anomalous LVA of aortic origin that receives high pulsatile flow is passing through the SG. Expansion of its (VA) wall during high pulsatile flow may compress the sympathetic fibres producing signs of Horner's syndrome. To the best of our knowledge, this is the first reported case describing RVA and LVA passing through the SG before entering into their respective foramen transversarium. Loh et al. [8] has reported that SG block relieves certain abnormal painful states whether the lesion causing them is peripheral or central. In a systemic review of complications associated with stellate ganglion nerve block (SGNB), one death due to massive haematoma leading to airway obstruction has been reported by Goel et al. [5]. In present case, as the VA is passing through the SG there is a high chance of it getting punctured during SGNB technique. Although this procedure is performed under landmark based technique, fluoroscopy or ultrasound but still complications can occur (Fig. 3).

According to Kiray et al. [6], anterolateral approach to the cervical spine requires greater retraction of longus colli and longus capitis muscles which has greater risk of injury to CST. If a patient having variation where VA is passing through SG undergoes cervical spine surgery, there is a high chance of injury to CST and VA leading to haemorrhage. According to Tubbs et al. [18], VA makes a medial indentation at C2 as it travels through the transverse foramen. The authors classified the variations of VA cave which would be helpful to the surgeons during $C 2$ pedicle screw placement [18]. Our present case closely resembles the study done by Tardieu et al. [16], Woraputtaporn et al. [19] and Komiyama et al. [7] up to some extent because in our case, VA arising from aortic arch on the left side has a more medial course and it enters the fifth cervical transverse foramina (Fig. 1B, 3B) and its length is twice as long as the RVA but sign of arterial dissection in $\mathrm{V} 1$ segment was absent.

\section{CONCLUSIONS}

Therefore as VA and CST are in close relation to each other in cervical region, this rare anomaly should be kept in mind by the anaesthetist during SGNB otherwise it can lead to haemorrhage. Surgeons should be aware of this rare anomaly while performing any surgery in cervical region because lack of knowledge of anatomy of sympathetic trunk and the surrounding anatomy may complicate surgical procedures on the cervical spine. It is of critical importance for preoperative imaging (computed tomography or magnetic resonance imaging) to be reviewed and course of VA and structure through which it is passing be assessed to prevent iatrogenic injury to VA and its potentially devastating effect.

\section{Acknowledgements}

We are thankful to Dr. Ashutosh Kumar, Department of Anatomy, AllMS Patna for his help in preparing this case report.

\section{REFERENCES}

1. Cheng $M$, Xiaodong $X$, Wang $C$, et al. Two anatomic variations of the vertebral artery in four patients. Ann Vasc Surg. 2009; 23(5): 689.e1-689.e5, doi: 10.1016/j. avsg.2009.06.007, indexed in Pubmed: 19747615.

2. Dzierżanowski J, Szarmach A, Baścik B, et al. Intracranial region of the vertebral artery: morphometric study in the context of clinical usefulness. Folia Morphol. 2017; 76(3): 379-387, doi: 10.5603/FM.a2017.0021, indexed in Pubmed: 28281722.

3. Dutta AK. Principles of general anatomy. 2nd Ed. Kolkata: K P Basu Publishing Co 2015.

4. Elgueta MF, Ortiz Jimenez J, Wang NN, et al. Anatomical variations of the vertebral artery in the upper cervical spine: clinical relevance for procedures targeting the C1/C2 and C2/C3 joints. Reg Anesth Pain Med. 2018; 43(4): 367-371, doi: 10.1097/AAP.0000000000000734, indexed in Pubmed: 29346229.

5. Goel V, Patwardhan A, Ibrahim M, et al. Complications associated with stellate ganglion nerve block: a systematic review. Reg Anesth Pain Med. 2019; 44(6): 669-678, doi: 10.1136/rapm-2018-100127.

6. Kiray A, Arman C, Naderi S, et al. Surgical anatomy of the cervical sympathetic trunk. Clin Anat. 2005; 18(3): 179-185, doi: 10.1002/ca.20055, indexed in Pubmed: 15768422

7. Komiyama $\mathrm{M}$, Morikawa $\mathrm{T}$, Nakajima $\mathrm{H}$, et al. High incidence of arterial dissection associated with left vertebral artery of aortic origin. Neurol Med Chir (Tokyo). 2001; 41(1): 8-11; discussion 11, doi: 10.2176/nmc.41.8, indexed in Pubmed: 11218642.

8. Loh L, Nathan PW, Schott GD. Pain due to lesions of central nervous system removed by sympathetic block. Br Med J (Clin Res Ed). 1981; 282(6269): 1026-1028, doi: 10.1136/ bmj.282.6269.1026, indexed in Pubmed: 6783230.

9. Patil ST, Meshram MM, Kamdi NY, et al. Study on branching pattern of aortic arch in Indian. Anat Cell Biol. 2012; 45(3): 203-206, doi: 10.5115/acb.2012.45.3.203, indexed in Pubmed: 23094209. 
10. Polguj $M$, Jędrzejewski $K$, Topol $M$, et al. Duplication of the left vertebral artery in a patient with dissection of the right internal carotid artery and Ehlers-Danlos syndrome: case report and review of the literature. Anat Sci Int. 2013; 88(2): 109-114, doi: 10.1007/s12565-012-0152-z, indexed in Pubmed: 22956231.

11. Polguj $M$, Podgórski M, Jędrzejewski K, et al. Fenestration and duplication of the vertebral artery: the anatomical and clinical points of view. Clin Anat. 2013; 26(8): 933-943, doi: 10.1002/ca.22231, indexed in Pubmed: 23553773.

12. Shoja MM, Tubbs RS, Khaki AA, et al. A rare variation of the vertebral artery. Folia Morphol. 2006; 65(2): 167-170.

13. Raval JD, Jadav HR. Anatomical study of variation of vertebral artery entering the foramen transversarium of cervical vertebrae. Natl J Med Res. 2012; 2: 199-201.

14. Sinnatamby CS. Last's anatomy: regional and applied. 12 th ed. Churchill Livingstone, Edinburg 2011.

15. Standring S. Gray's anatomy: the anatomical basis of clinical practice. 40th ed. Churchill Livingstone, London 2008.
16. Tardieu GG, Edwards B, Alonso F, et al. Aortic arch origin of the left vertebral artery: An Anatomical and Radiological Study with Significance for Avoiding Complications with Anterior Approaches to the Cervical Spine. Clin Anat. 2017; 30(6): 811-816, doi: 10.1002/ca.22923, indexed in Pubmed: 28547783.

17. Tetiker H, Cimen M, Koşar MI. Fenestration of the vertebral artery: case presentation. Folia Morphol. 2014; 73(1): 84-86, doi: 10.5603/FM.2014.0012, indexed in Pubmed: 24590528.

18. Tubbs RS, Granger A, Fisahn C, et al. The Vertebral Artery Cave at C2: Anatomic Study with Application to C2 Pedicle Screw Placement. World Neurosurg. 2016; 94: 368-374, doi: 10.1016/j.wneu.2016.07.037, indexed in Pubmed: 27443230.

19. Woraputtaporn W, Ananteerakul T, lamsaard S, et al. Incidence of vertebral artery of aortic arch origin, its level of entry into transverse foramen, length, diameter and clinical significance. Anat Sci Int. 2019; 94(4): 275-279, doi: 10.1007/ s12565-019-00482-6, indexed in Pubmed: 30806941. 